Reprod. Nutr. Dévelop., 1988, 28 (2 A), 253-256.

\title{
Effets du GRF 1-29 chez l'agneau normal et hypotrophique
}

P. PASTOUREAU $\left({ }^{1},{ }^{3}\right)$, B. BARENTON $\left({ }^{2}\right)$, Monique BLANCHARD $\left({ }^{2}\right)$, G. BOIVIN $\left({ }^{3}\right)$, J. CHARRIER ( $\left.{ }^{2}\right)$, J.-P. DULOR $\left({ }^{2}\right)$, M. THERIEZ $\left({ }^{1}\right)$

(1) Laboratoire de la Production ovine, I.N.R.A. Theix 63122 Ceyrat, France.

(2) Station de Physiologie animale, I.N.R.A.-E.N.S.A., 34060 Montpellier, Cedex.

(3) INSERM, Unité 234,

Faculté A. Carrel, 69008 Lyon.

Summary. Effects of GRF 1.29 in normal and hypotrophic lambs.

Injections of hGRF rapidly stimulated $\mathrm{GH}$ secretion in lamb and this response was significantly higher in hypotrophic than in normal lambs. The treatment had an effect on body composition reducing the retention of lipids and the energy level and increasing calcium and phosphorus content. The results showed that GRF affected both metabolism and skeletal growth of ruminants, stimulating specially the production of $\mathrm{Sm}-\mathrm{C}$ and proliferation of chondrocytes.

\section{Introduction.}

II est bien admis que l'hormone de croissance $(\mathrm{GH})$ joue un rôle majeur dans le contrôle hormonal de la croissance, induisant notamment la synthèse de somatomédine $\mathrm{C}(\mathrm{Sm}-\mathrm{C})$ ou stimulant directement la prolifération des chondrocytes (Isaksson et al., 1982). Chez l'agneau, nous avons précédemment montré qu'une injection intraveineuse de GRF 1-29 (Barenton et al., 1987) provoque une augmentation rapide de la sécrétion de GH. Par ailleurs, l'existence d'agneaux hypotrophiques (poids réduit à la naissance : $<2,5 \mathrm{~kg}$ ) dont le potentiel de croissance semble altéré comparativement à des agneaux normaux (poids à la naissance $>4,0 \mathrm{~kg}$ ) nous a conduit à étudier les effets d'un traitement à long terme au GRF 1-29 sur, les teneurs plasmatiques en GH et Sm-C, la croissance osseuse, les performances zootechniques et la composition corporelle d'agneaux normaux et hypotrophiques à la naissance.

\section{Matériel et méthodes.}

Quarante six agneaux mâles ont été répartis en deux groupes suivant leur poids à la naissance (PN) : 24 agneaux normaux ( $P N=4,3 \pm 0,2 \mathrm{~kg}$ ) et 22 agneaux hypotrophiques ( $P N=2,4 \pm 0,2 \mathrm{~kg}$ ). Dans chaque groupe, la moitié 
des agneaux reçurent de la naissance à l'abattage (à 45 ou à 90 jours) 2 injections quotidiennes sous-cutanées de GRF 1-29 $(8 \mu \mathrm{g} / \mathrm{kg}$ de poids vif ; don de SanofiRecherche). Durant le traitement, des contrôles individuels du poids vif furent pratiqués régulièrement. En début et en fin d'expérience, ont été mesurés les taux plasmatiques en GH (RIA double anticorps oGH-S-5 et antisérum anti-oGH fourni par le National Pituitary Program, Bethesda) et en Sm-C après extraction des échantillons à I'acide/alcool (RIA double anticorps, I'antigène étant $S \mathrm{~m}-\mathrm{C}$ recombiné de Amgen et I'antisérum fourni par le National Pituitary Program). A l'abattage le métacarpe gauche fut prélevé, puis une étude histomorphométrique fut réalisée sur des sections non décalcifiées de la zone de la plaque de croissance des métacarpes. Après l'abattage tout l'animal fut broyé pour l'analyse de la composition chimique (lipides, calcium, phosphore).

\section{Résultats et discussion.}

Chez les agneaux traités, après l'injection de GRF, on observe une stimulation rapide de la sécrétion de $\mathrm{GH}$ dès la naissance, à 45 jours et à 90 jours (fig. 1) ; par ailleurs, la durée et l'amplitude de la sécrétion de $\mathrm{GH}$ sont toujours significativement plus importantes chez les agneaux hypotrophiques que chez les agneaux normaux. Au bout de 45 jours, le GRF induit une augmentation des teneurs plasmatiques en Sm-C chez les agneaux hypotrophiques (312 \pm 16 vs $234 \pm 23 \mathrm{ng}$ / $\mathrm{ml}$ pour les agneaux normaux) $\left({ }^{*}\right)$. Cependant cet effet $\mathrm{n}^{\prime} \mathrm{a}$ pas été retrouvé à l'issue de la période de 90 jours. Les résultats d'histomorphométrie osseuse vont dans le même sens que les résultats biochimiques. Conformément aux résultats publiés par Thorngren et Hansson (1973b, 1974) et Lindahl et al. (1986), le GRF a stimulé la prolifération des chondrocytes dans la plaque de croissance, dès
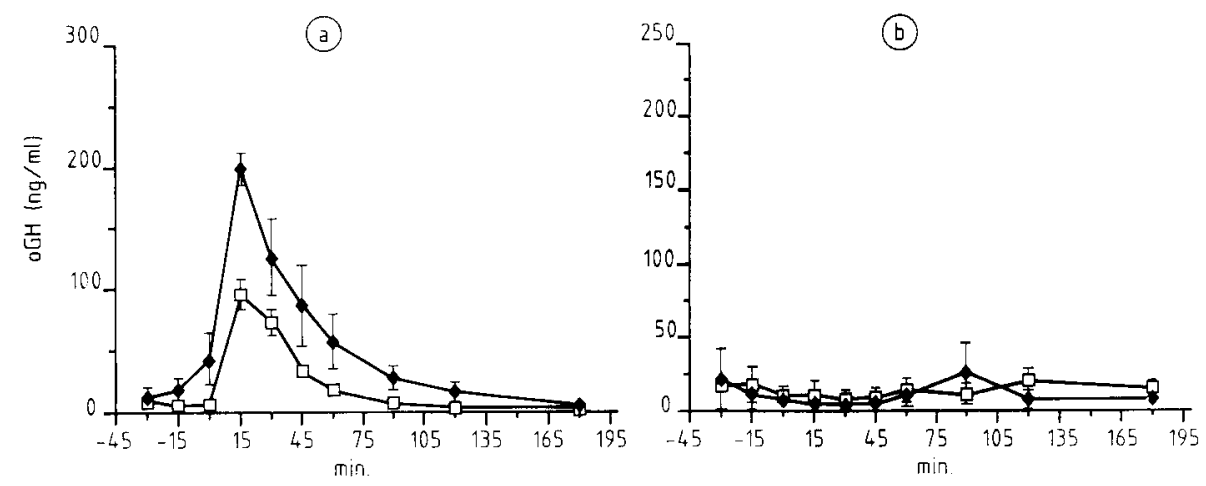

FIG. 1. - Profil de sécrétion d'oGH en début de traitement par $/ e$ GRF $(8 \mu \mathrm{g} / \mathrm{kg}$ de poids vif $)$ chez les agneaux normaux et hypotrophiques à la naissance. a : animaux traités ; b : témoins ; : normaux ; $\checkmark$ : hypotrophiques.

(*) Moyenne \pm sem. 
45 jours chez les agneaux hypotrophiques et dans les 2 groupes d'agneaux au bout de 90 jours de traitement (tabl. 1). L'absence d'effet du GRF sur les performances zootechniques des animaux pourrait s'expliquer par la brièveté de la réponse sous forme de sécrétion de $\mathrm{GH}$.

En revanche, les résultats de composition chimique d'agneaux broyés après l abattage font apparaître des effets significatifs du GRF (tabl. 2). Au bout de 45 jours, le traitement a significativement réduit les quantités de lipides et d'éner-

\section{TABLEAU 1}

Effet d'un traitement journalier par le GRF $(2 \times 8 \mu \mathrm{g} / \mathrm{kg})$ durant 45 ou 90 jours chez l'agneau normal ou hypotrophique. Résultats histomorphométriques.

\begin{tabular}{|c|c|c|c|c|}
\hline $\begin{array}{l}\text { Durée du } \\
\text { traitemen } \\
\text { (jour) }\end{array}$ & $\begin{array}{ll}\text { uu } & \text { Groupes } \\
\text { nt } & (n)\end{array}$ & $\begin{array}{c}\text { Taux de croissance } \\
\text { longitudinale (a) } \\
(\mu \mathrm{m} / \text { jour })\end{array}$ & $\begin{array}{c}\text { Taille des cellules } \\
\text { dégénératives }(\mathrm{b}) \\
(\mu \mathrm{m})\end{array}$ & $\begin{array}{l}\text { Taux de production } \\
\text { cellulaire }(a: b) \\
\text { (nb de cellules/jour) }\end{array}$ \\
\hline \multirow{4}{*}{ t } & $\begin{array}{l}\text { Normaux } \\
\text { témoins }(6) \\
\text { Normaux }\end{array}$ & $283 \pm 12$ & $28 \pm 0,8$ & $10,1 \pm 0,3$ \\
\hline & GRF (6) & $246 \pm 17$ & $28 \pm 0,8$ & $8,7 \pm 0,5^{*}$ \\
\hline & $\begin{array}{l}\text { Hypotrophiques } \\
\text { témoins }(5) \\
\text { Hypotrophiques }\end{array}$ & $254 \pm 22$ & $31 \pm 0,3$ & $8,2 \pm 0,7$ \\
\hline & GRF (5) & $274 \pm 12$ & $28 \pm 0,7^{*}$ & $9,9 \pm 0,6$ \\
\hline \multirow{4}{*}{90} & $\begin{array}{l}\text { Normaux } \\
\text { témoins (6) } \\
\text { Normaux }\end{array}$ & $177 \pm 10$ & $27 \pm 0,3$ & $6,7 \pm 0,4$ \\
\hline & GRF (6) & $196 \pm 11$ & $26 \pm 0,6$ & $7,5 \pm 0,5$ \\
\hline & $\begin{array}{l}\text { Hypotrophiques } \\
\text { témoins }(6) \\
\text { Hypotrophiques }\end{array}$ & $161 \pm 16$ & $26 \pm 1,2$ & $6,2 \pm 0,4$ \\
\hline & GRF (6) & $180 \pm 11$ & $24 \pm 0,8^{*}$ & $7,7 \pm 0,8^{*}$ \\
\hline
\end{tabular}

Moyennes \pm sem pour le nombre d'animaux $(n)$ dans chaque groupe.

* : vs témoins correspondants $\mathrm{p}<0,05$.

TABLEAU 2

Effet du traitement GRF sur la composition chimique des agneaux après broyage à 45 et à 90 jours. Pésultats exprimés en termes de différence de quantités brutes : traités - témoins.

\begin{tabular}{ccccc}
\hline $\begin{array}{c}\text { Durée du } \\
\text { traitement } \\
\text { (jour) }\end{array}$ & $\begin{array}{c}\text { Lipides } \\
(\mathrm{g})\end{array}$ & $\begin{array}{c}\text { Energie } \\
(\mathrm{kcal})\end{array}$ & $\begin{array}{c}\text { Calcium } \\
(\mathrm{g})\end{array}$ & $\begin{array}{c}\text { Phosphore } \\
(\mathbf{g})\end{array}$ \\
\hline 45 & $-288^{*}$ & $-5590^{*}$ & $+9,4$ & $+13,6^{*}$ \\
\hline 90 & +50 & +1504 & +20 & +18 \\
\hline
\end{tabular}

* $: p<0,01$. 
gie fixées par l'animal et augmenté la quantité de phosphore. Par ailleurs, il est intéressant de noter l'augmentation simultanée des quantités de calcium (non significative) et de phosphore fixées par les animaux après 45 jours et 90 jours de traitement ; ceci tendrait à prouver un effet plus ciblé du GRF sur le squelette.

12e Réunion du groupe Développement I.N.R.A.

Montpellier, 28-30 mai 1986.

Recu en septembre 1987.

Accepté en octobre 1987.

\section{Références}

BARENTON B., DUCLOS M., DIAZ J., DELÉTANG F., DULOR J.-P., BLANCHARD M., CHARRIER J., 1987. Characteristics of growth hormone response to the administration of growth hormone releasing hormone (GRF) in the lamb. Reprod. Nutr. Dévelop., 27, 491-500.

ISAKSSON O. G. P., JANSSON J. O., GAUSE I. A. M., 1982. Growth hormone stimulates longitudinal bone growth directly. Science, 216, 1237.

LINDAHL A., ISGAARD J., NILSSON A., ISAKSSON O. G. P., 1986. Growth hormone potentiates colony formation of epiphyseal chondrocytes in suspension culture. EndocrinologV, 118, 1843-1848.

THORNGREN K. G., HANSSON L. I., 1973b. Effect of thyroxine and growth hormone on longitu dinal bone growth in the hypophysectomized rat. Acta endocrinol., 74, 24-40.

THORNGREN K. G., HANSSON L. I., 1974. Effect of withdrawal of growth hormone administration on longitudinal bone growth in the hypophysectomized rat. Acta endocrinol., 75, 11-23. 